PROCEEDINGS OF THE

AMERICAN MATHEMATICAL SOCIETY

Volume 133, Number 9, Pages 2657-2660

S 0002-9939(05)07983-9

Article electronically published on March 22, 2005

\title{
FENCHEL DUALITY, FITZPATRICK FUNCTIONS AND THE KIRSZBRAUN-VALENTINE EXTENSION THEOREM
}

\author{
SIMEON REICH AND STEPHEN SIMONS
}

(Communicated by Jonathan M. Borwein)

\begin{abstract}
We present a new proof of the classical Kirszbraun-Valentine extension theorem. Our proof is based on the Fenchel duality theorem from convex analysis and an analog for nonexpansive mappings of the Fitzpatrick function from monotone operator theory.
\end{abstract}

Let $H$ be a real Hilbert space, let $D$ be a nonempty subset of $H$ and let $T: D \mapsto H$ be nonexpansive. According to the Kirszbraun-Valentine extension theorem ([5], 12]), there is a nonexpansive extension of $T$ to the whole of $H$. Proofs of this classical result, as well as related information, can be found in, for instance, [6], 10], [3], 13] and [1]. The purpose of this note is to present a new proof of the Kirszbraun-Valentine extension theorem, which we state as Theorem 5 below. Our proof is based on the Fenchel duality theorem from convex analysis (see Theorem 1 below) and an analog for nonexpansive maps of the Fitzpatrick function from monotone multifunction theory (see [4] or [1] — in the context of monotone multifunctions, related functions appear in [2] and [8]). We start off by stating Rockafellar's version of the Fenchel duality theorem (see [9, Theorem 1, pp. 82-83]). We recall that if $f: E \mapsto(-\infty, \infty]$ is convex, then the Fenchel conjugate, $f^{*}$, of $f$ is the function on the dual, $E^{*}$, of $E$ defined by $f^{*}\left(x^{*}\right)=\sup _{E}\left[x^{*}-f\right]$.

Theorem 1. Let $E$ be a nonzero normed space, let $f, g: E \mapsto(-\infty, \infty]$ be proper and convex, let $g$ be finite and continuous at a point where $f$ is finite, and let $f+g \geq 0$ on $E$. Then there exists $x^{*} \in E^{*}$ such that $f^{*}\left(x^{*}\right)+g^{*}\left(-x^{*}\right) \leq 0$.

Definition 2. Let $D$ be a nonempty subset of a Hilbert space $H$ and let $T: D \mapsto H$. The map $T$ is said to be nonexpansive if $x, y \in D \Longrightarrow\|T(x)-T(y)\| \leq\|x-y\|$. We say that $T$ is maximally nonexpansive if $T$ is nonexpansive, and there is no nonexpansive extension of $T$ to a proper superset of $D$.

Lemma 3. Let $T: D \mapsto H$ be maximally nonexpansive, and define $\chi: H \times H \mapsto$ $(-\infty, \infty]$ by

$$
\chi(p, q):=\sup _{d \in D}\left[\|q-T(d)\|^{2}-\|p-d\|^{2}\right] .
$$

Let $G(T)$ be the graph of $T,\{(p, T(p)): p \in D\}$. Then:

(a) $\chi \geq 0$ on $H \times H$.

(b) $\chi(p, q)=0 \Longleftrightarrow(p, q) \in G(T)$.

Received by the editors April 21, 2004.

2000 Mathematics Subject Classification. Primary 46C05, 47H09; Secondary 46N10.

(C) 2005 American Mathematical Society 
Proof. (a) If $(p, q) \in(H \backslash D) \times H$, then we can extend $T$ to a function $\widetilde{T}$ on $D \cup\{p\}$ by defining $\widetilde{T}(p)=q$. Since $T$ is maximally nonexpansive, $\widetilde{T}$ is not nonexpansive on $D \cup\{p\}$, from which it easily follows that there exists $d \in D$ such that $\| \widetilde{T}(p)-$ $\widetilde{T}(d)\|>\| p-d \|$, that is to say, $\|q-T(d)\|>\|p-d\|$. Consequently, $\chi(p, q)>0$. If, on the other hand, $(p, q) \in D \times H$, then $\chi(p, q) \geq\|q-T(p)\|^{2}-\|p-p\|^{2}=$ $\|q-T(p)\|^{2} \geq 0$. This completes the proof of (a).

(b) If $(p, q) \in G(T)$, then, since $T$ is nonexpansive on $D$, for all $d \in D$,

$$
\|q-T(d)\|^{2}-\|p-d\|^{2}=\|T(p)-T(d)\|^{2}-\|p-d\|^{2} \leq 0,
$$

while $\|q-T(p)\|^{2}-\|p-p\|^{2}=0-0=0$, which shows that $\chi(p, q)=0$. Conversely, if $\chi(p, q)=0$, then the proof of (a) shows first that $(p, q) \notin(H \backslash D) \times H$, from which $(p, q) \in D \times H$, and then that $\|q-T(p)\|^{2}=0$, from which $(p, q) \in G(T)$, completing the proof of (b).

Lemma 4. Let $T: D \mapsto H$ be maximally nonexpansive and define $\varphi$ : $H \times H \mapsto$ $(-\infty, \infty]$ by

$$
\varphi\left(x, x^{*}\right):=\frac{1}{4} \chi\left(x+x^{*}, x-x^{*}\right)+\left\langle x, x^{*}\right\rangle .
$$

Define the norm of $H \times H$ by $\left\|\left(x, x^{*}\right)\right\|:=\sqrt{\|x\|^{2}+\left\|x^{*}\right\|^{2}}$. Then:

(a) For all $\left(x, x^{*}\right) \in H \times H$,

$$
\varphi\left(x, x^{*}\right)=\frac{1}{4} \sup _{d \in D}\left[2\langle x, d-T(d)\rangle+2\left\langle x^{*}, d+T(d)\right\rangle+\|T(d)\|^{2}-\|d\|^{2}\right] .
$$

(b) For all $d \in D, \varphi\left(\frac{1}{2}(d+T(d)), \frac{1}{2}(d-T(d))\right)=\frac{1}{4}\|d\|^{2}-\frac{1}{4}\|T(d)\|^{2}$.

(c) $\varphi$ is proper, convex and lower semicontinuous.

(d) For all $\left(z^{*}, z\right) \in H \times H, \varphi^{*}\left(z^{*}, z\right) \geq \varphi\left(z, z^{*}\right)$.

(e) $D \ni 0$.

(f) $D=H$.

Proof. (a) This follows from the observation that, for all $\left(x, x^{*}\right) \in H \times H$ and $d \in D$,

$$
\begin{aligned}
\| x & -x^{*}-T(d)\left\|^{2}-\right\| x+x^{*}-d \|^{2} \\
& =\left\|x-x^{*}\right\|^{2}-2\left\langle x-x^{*}, T(d)\right\rangle+\|T(d)\|^{2}-\left\|x+x^{*}\right\|^{2}+2\left\langle x+x^{*}, d\right\rangle-\|d\|^{2} \\
& =-4\left\langle x, x^{*}\right\rangle+2\langle x, d-T(d)\rangle+2\left\langle x^{*}, d+T(d)\right\rangle+\|T(d)\|^{2}-\|d\|^{2} .
\end{aligned}
$$

(b) This follows since, for all $d \in D$, Lemma 3 (b) gives

$$
\begin{aligned}
\varphi\left(\frac{1}{2}(d+T(d)), \frac{1}{2}(d-T(d))\right) & =\frac{1}{4} \chi(d, T(d))+\frac{1}{4}\langle d+T(d), d-T(d)\rangle \\
& =\frac{1}{4}\langle d+T(d), d-T(d)\rangle=\frac{1}{4}\|d\|^{2}-\frac{1}{4}\|T(d)\|^{2} .
\end{aligned}
$$

(c) This is immediate from (a) and (b).

(d) We have from (b) that

$\varphi^{*}\left(z^{*}, z\right)$

$$
\begin{aligned}
& :=\sup _{\left(x, x^{*}\right) \in H \times H}\left[\left\langle x, z^{*}\right\rangle+\left\langle x^{*}, z\right\rangle-\varphi\left(x, x^{*}\right)\right] \\
& \geq \sup _{d \in D}\left[\left\langle\frac{1}{2}(d+T(d)), z^{*}\right\rangle+\left\langle\frac{1}{2}(d-T(d)), z\right\rangle-\varphi\left(\frac{1}{2}(d+T(d)), \frac{1}{2}(d-T(d))\right)\right] \\
& =\sup _{d \in D}\left[\left\langle\frac{1}{2}(d+T(d)), z^{*}\right\rangle+\left\langle\frac{1}{2}(d-T(d)), z\right\rangle-\frac{1}{4}\|d\|^{2}+\frac{1}{4}\|T(d)\|^{2}\right] \\
& =\frac{1}{4} \sup _{d \in D}\left[2\left\langle d+T(d), z^{*}\right\rangle+2\langle d-T(d), z\rangle-\|d\|^{2}+\|T(d)\|^{2}\right]
\end{aligned}
$$

and the result now follows from (a). 
(e) For all $\left(x, x^{*}\right) \in H \times H$,

$$
\varphi\left(x, x^{*}\right)+\frac{1}{2}\left\|\left(x, x^{*}\right)\right\|^{2}=\frac{1}{4} \chi\left(x+x^{*}, x-x^{*}\right)+\left\langle x, x^{*}\right\rangle+\frac{1}{2}\left\|\left(x, x^{*}\right)\right\|^{2} .
$$

Thus, from Lemma 3(a),

$$
\left\{\begin{aligned}
\varphi\left(x, x^{*}\right)+\frac{1}{2}\left\|\left(x, x^{*}\right)\right\|^{2} & \geq\left\langle x, x^{*}\right\rangle+\frac{1}{2}\left\|\left(x, x^{*}\right)\right\|^{2} \\
& \geq \frac{1}{2}\|x\|^{2}+\frac{1}{2}\left\|x^{*}\right\|^{2}-\|x\|\left\|x^{*}\right\| \geq 0 .
\end{aligned}\right.
$$

Part (c) and Theorem 1 now give us an element $\left(z^{*}, z\right)$ of $(H \times H)^{*}=H \times H$ such that $\varphi^{*}\left(z^{*}, z\right)+\frac{1}{2}\left\|\left(-z^{*},-z\right)\right\|^{2} \leq 0$. Part (d) and (4.1) imply, in turn, that $\varphi\left(z, z^{*}\right)+\frac{1}{2}\left\|\left(z, z^{*}\right)\right\|^{2}=0$, that is to say,

$$
\frac{1}{4} \chi\left(z+z^{*}, z-z^{*}\right)+\left\langle z, z^{*}\right\rangle+\frac{1}{2}\left\|\left(z, z^{*}\right)\right\|^{2}=0,
$$

which can be rewritten as

$$
\frac{1}{4} \chi\left(z+z^{*}, z-z^{*}\right)+\frac{1}{2}\left\|z+z^{*}\right\|^{2}=0 .
$$

It is clear from Lemma 3(a) that $\left\|z+z^{*}\right\|^{2}=0$, that is to say $z+z^{*}=0$, and then $\chi\left(0, z-z^{*}\right)=0$. Lemma 3(b) now gives $\left(0, z-z^{*}\right) \in G(T)$, from which (e) follows.

(f) This is immediate from (e) and a simple translation argument.

Theorem 5. Let $T: D \mapsto H$ be nonexpansive. Then there is a nonexpansive extension of $T$ to $H$.

Proof. Zorn's lemma easily gives that there is a maximal nonexpansive extension of $T$. The result now follows from Lemma 4(f).

Remark 6 . We note the fact that we have actually proved in Lemma 4(e) that

$$
\min _{\left(x, x^{*}\right) \in H \times H}\left[\phi\left(x, x^{*}\right)+\frac{1}{2}\left\|\left(x, x^{*}\right)\right\|^{2}\right]=0 .
$$

Remark 7. The proof of Theorem 1 uses the Eidelheit separation theorem in $E \times \mathbb{R}$. It would be interesting to find a more direct proof for the special case we need for Lemma $4(\mathrm{e})$. This can be stated without conjugate functions in the following way: if $f: H \mapsto(-\infty, \infty]$ is convex and, for all $x \in H, f(x)+\frac{1}{2}\|x\|^{2} \geq 0$, then there exists $y \in H$ such that, for all $x \in H, f(x) \geq\langle x, y\rangle+\frac{1}{2}\|y\|^{2}$.

Remark 8. We note that, in contrast with other proofs, the proof of Theorem 5 given here does not use any compactness arguments. We also remark that it was observed in [7] that Theorem 5 is equivalent to Minty's theorem on maximal monotone multifunctions.

Remark 9. We show in this remark how Theorem 5 can be bootstrapped to give a similar result for maps from one Hilbert space into another. Let $H_{1}$ and $H_{2}$ be Hilbert spaces, $\emptyset \neq D_{1} \subset H_{1}$ and $U: D_{1} \mapsto H_{2}$ be nonexpansive. Define $T: D_{1} \times H_{2} \mapsto H_{1} \times H_{2}$ by $T\left(x_{1}, x_{2}\right):=\left(0, U\left(x_{1}\right)\right)$. Then $T$ is nonexpansive. Theorem 5 gives a nonexpansive $\widetilde{T}: H_{1} \times H_{2} \mapsto H_{1} \times H_{2}$ such that $\left.\widetilde{T}\right|_{D_{1} \times H_{2}}=T$. If $\mathrm{pr}_{2}$ is the projection from $H_{1} \times H_{2}$ onto $H_{2}$, then the map $\widetilde{U}: H_{1} \mapsto H_{2}$ defined by $\widetilde{U}\left(x_{1}\right):=\operatorname{pr}_{2} \widetilde{T}\left(x_{1}, 0\right)$ is a nonexpansive extension of $U$. 
Remark 10. If $T$ is maximally nonexpansive, then the operator $S$ defined by

$$
G(S):=\{((p-q) / 2,(p+q) / 2):(p, q) \in G(T)\}
$$

is maximal monotone. The Fitzpatrick function of $S, \varphi_{S}$, is then given by the formula $\varphi_{S}\left(x, x^{*}\right):=\sup _{\left(s, s^{*}\right) \in G(S)}\left[\left\langle x, s^{*}\right\rangle+\left\langle s, x^{*}\right\rangle-\left\langle s, s^{*}\right\rangle\right]$. This turns out to be identical with $\varphi\left(x, x^{*}\right)$ as defined in Lemma 4.

\section{REFERENCES}

[1] Y. Benyamini and J. Lindenstrauss, Geometric nonlinear functional analysis, Amer. Math. Soc. Coll. Pubs. 48, Providence, RI, 2000. MR.1727673 (2001b:46001)

[2] H. Brezis and A. Haraux, Image d'une somme d'opérateurs monotones at applications, Israel J. Math. 23 (1976), 165-186. MR0399965 (53:3803)

[3] H. Federer, Geometric measure theory, Springer-Verlag, New York, 1969. MR0257325 (41:1976)

[4] S. P. Fitzpatrick, Representing monotone operators by convex functions, Workshop/ Miniconference on Functional Analysis and Optimization, Proc. Centre Math. Anal. Austral. Nat. Univ., vol. 20, Austral. Nat. Univ., Canberra, 1988, pp. 59-65. MR1009594 (90i:47054)

[5] M. D. Kirszbraun, Über die zusammenziehende und Lipschitzsche Transformationen, Fund. Math. 22 (1934), 77-108.

[6] E. J. Mickle, On the extension of a transformation, Bull. Amer. Math. Soc. 55 (1949), 160164. MR0029974(10:691b)

[7] S. Reich, Extension problems for accretive sets in Banach spaces, J. Functional Analysis 26 (1977), 378-395. MR0477893 (57:17393)

[8] S. Reich, The range of sums of accretive and monotone operators, J. Math. Anal. Appl. 68 (1979), 310-317. MR0531440 (80g:47060)

[9] R. T. Rockafellar, Extension of Fenchel's duality theorem for convex functions, Duke Math. J. 33 (1966), 81-89. MR0187062 (32:4517)

[10] I. J. Schoenberg, On a theorem of Kirzbraun and Valentine, Amer. Math. Monthly 60 (1953), 620-622. MR0058232 (15,341c)

[11] S. Simons and C. Zălinescu, Fenchel duality, Fitzpatrick functions and maximal monotonicity, J. Nonlinear Convex Anal., in press.

[12] F. A. Valentine, A Lipschitz condition preserving extension for a vector function, Amer. J. Math. 67 (1945), 83-93. MR0011702 (6:203e)

[13] J. H. Wells and L. R. Williams, Embeddings and extensions in analysis, Springer-Verlag, New York-Heidelberg, 1975. MR0461107 (57:1092)

Department of Mathematics, The Technion - Israel Institute of Technology, 32000 HAIFA, IsRAel

E-mail address: sreich@tx.technion.ac.il

Department of Mathematics, University of California, Santa Barbara, California 93106-3080

E-mail address: simons@math.ucsb.edu 